\title{
Mamíferos medianos y grandes asociados al bosque tropical seco del centro de México
}

Luz Adriana Pérez-Solano ${ }^{1 *}$, Matilde González ${ }^{2}$, Eva López-Tello ${ }^{3} \&$ Salvador Mandujano ${ }^{1}$

1. Red de Biología y Conservación de Vertebrados, Instituto de Ecología, A.C., Carretera antigua a Coatepec 351, El Haya, Xalapa 91070, Veracruz, México; adriana.perez.s@outlook.com, salvador.mandujano@inecol.mx

2. Instituto Tecnológico de Úrsulo Galván, Secretaria de Educación Pública, Úrsulo Galván, Veracruz, México; bimati_1328@outlook.com

3. Posgrado en Neuroetología, Instituto de Neuroetología, Universidad Veracruzana, Av. Dr. Luis Castelazo s/n, Fracc. Industrial de las Ánimas, Xalapa 91190, Veracruz, México; evaltm20@gmail.com * Correspondencia

Recibido 12-II-2018. Corregido 28-V-2018. Aceptado 20-VI-2018.

\begin{abstract}
Medium and large mammals associated with the tropical dry forest of central Mexico. The tropical dry forest is one of the most endangered ecosystems in the world, mainly due to deforestation and fragmentation. In Mexico more than half of its surface has disappeared, affecting biodiversity. The objective of this study was to compare the richness, diversity, and relative abundance of medium and large mammals in two sites with different management and conservation status, and where tropical dry forest is present. We also sought to find out which variables of habitat and human influence are related to the presence of the most common species. The study sites were in Pastorias, Actopan, Veracruz and San Juan Bautista Cuicatlan, Oaxaca, Mexico. The first site is highly transformed by anthropogenic activities, while the second is within the protected natural area of the Tehuacan-Cuicatlan Biosphere Reserve (TCBR). In Pastorias 11 camera-traps were set up from september 2015 to september 2016; while in Cuicatlan 10 camera traps were set up from october 2013 to july 2014. The sampling effort was 3526 days/trap, which yielded 443 independent records for the first site, and 2690 days/trap that yielded 378 independent records for the second site. In each locality, 13 species of mammals were recorded. In particular, three of these species are endangered (Tamandua mexicana, Leopardus wiedii, and Lynx rufus) and two are under threat (Herpailurus yagouaroundi and Galictis vittata). The true diversity of medium and large mammals between communities was different. According to the relative abundance, index (RAI) the most abundant species are Odocoileus virginianus, Urocyon cinereoargenteus and Canis latrans. Regression analyses suggest that the variables of season, the cover of secondary vegetation and distance to the nearest human locality were related to the record of these species. Despite the anthropogenic pressure in Pastorias, tropical dry forest remnants still maintain high species richness, which is very similar to that of a site as preserved as Cuicatlan. However, the low RAIs value of several mammals may indicate that habitat quality could not maintain large population numbers. Therefore, it is necessary to develop programs to conserve this ecosystem. Rev. Biol. Trop. 66(3): 1232-1243. Epub 2018 September 01.
\end{abstract}

Key words: camera-trapping; species richness; relative abundance; Veracruz, Oaxaca.

El bosque tropical seco representa el 42 $\%$ de los ecosistemas tropicales alrededor del mundo, el $78 \%$ de esta superficie ha sido modificado por actividades humanas, lo que lo ha convertido en uno de los más amenazados a nivel mundial (Miles et al., 2006; Ceballos \& Valenzuela, 2010). En México es el ecosistema tropical mejor representado debido a su extensión (Rzedowski, 1978; Dirzo \& Ceballos, 2010) y alberga una gran riqueza de vertebrados terrestres, en particular, el $35 \%$ de las especies de mamíferos reportadas en todo el país (Ceballos \& García, 1995; Ceballos \& Valenzuela, 2010). Sin embargo, se deforestan alrededor de 300000 hectáreas por año, tres veces más que la tasa de deforestación estimada 
para los bosques de coníferas y 1.5 veces más que para el bosque tropical perennifolio (Balvanera, Islas, Aguirre, \& Quijas, 2000).

La Reserva de la Biosfera de TehuacánCuicatlán (RBTC) ubicada al noroeste del estado de Oaxaca y sureste del estado de Puebla, es un sitio de interés para la conservación debido a la diversidad de especies que habitan, y donde el bosque tropical seco abarca gran parte de su extensión (CONANP, 2004). En la RBTC los estudios relacionados con la comunidad de mamíferos medianos y grandes se han enfocado principalmente en listados de especies (Briones-Salas, 2000; Dávila et al., 2002), nuevos registros de distribución (Bote1lo, Illoldi-Rangel, Linaje, \& Sánchez-Cordero, 2006a; Botello et al., 2006b; Botello et al., 2013), y en menor cantidad con aspectos poblacionales y ecológicos (Ortíz-García, RamosRobles, Pérez-Solano, \& Mandujano, 2012; Yañez-Arenas, Martínez-Meyer, Mandujano, \& Rojas-Soto, 2012; Cruz-Jácome, López-Tello, Delfín-Alfonso, \& Mandujano, 2015).

En contraste, en Veracruz el bosque tropical seco se encuentra altamente fragmentado $\mathrm{y}$ algunas regiones han sido transformadas para uso ganadero y agropecuario (CastilloCampos, Avendaño, \& Medina, 2011). Un claro ejemplo de esto es al centro del estado en la localidad de Pastorías, municipio de Actopan, sitio altamente heterogéneo donde se presentan pequeños remanentes de bosque tropical seco rodeados por parches de uso agrícola y vegetación secundaria, esta localidad no se encuentra dentro de un área natural protegida (ANP). En el centro de Veracruz existe un vacío informativo sobre las poblaciones de mamíferos que habitan en el bosque tropical seco, los estudios consisten principalmente en listados de especies (Morales-Mávil \& Guzmán-Guzmán, 1994; González-Romero \& Lara-López, 2006) realizados en la reserva privada "Centro de Investigaciones Costeras La Mancha", que forma parte de la ANP "La Mancha el Llano" (Moreno-Casasola, 2006).

Los mamíferos de talla media y grande se consideran como un grupo importante para monitorear la biodiversidad y el estado de integridad ecológica de un sitio (Baillie et al., 2008; Ahumada et al., 2011), por lo que estimar la riqueza de especies y la abundancia relativa es una manera de expresar la diversidad biológica de un sitio (Moreno, 2001). En este sentido, mediante el foto-trampeo en este trabajo se comparó la riqueza, diversidad y abundancia relativa de la comunidad de mamíferos medianos y grandes de dos sitios dominados por el bosque tropical seco, pero con características diferentes de manejo y conservación. Se buscó también conocer las variables del hábitat y de influencia humana que se relacionan con la presencia de las especies de mamíferos más comunes. Este trabajo es relevante para el desarrollo de planes de manejo y conservación de este ecosistema y de la fauna que alberga.

\section{MATERIALES Y MÉTODOS}

Sitios de estudio: Se trabajó en dos localidades: Pastorías, municipio de Actopan, Veracruz y San Juan Bautista Cuicatlán, Oaxaca, México. Pastorías se ubica en la zona centro del estado de Veracruz, entre las coordenadas 19³3' 52' N \& - 96³4'14” W, a $260 \mathrm{msnm}$, presenta un clima cálido subhúmedo con lluvias en verano, temperatura anual de $20-26{ }^{\circ} \mathrm{C}$ y precipitación anual de 1100 a $1300 \mathrm{~mm}$. Los principales tipos de vegetación son el bosque tropical seco y pastizal inducido. Históricamente el uso del suelo ha sido para producción ganadera y agropecuaria, como cultivo de caña y mango (INEGI, 2009; INAFED, 2010). San Juan Bautista Cuicatlán está ubicado entre las coordenadas $17^{\circ} 48^{\prime} \mathrm{N} \& 96^{\circ} 57^{\prime} \mathrm{W}$, a una altitud de $620 \mathrm{msnm}$, forma parte de La cañada Oaxaqueña y se localiza en la zona sur de la Reserva de la Biosfera de Tehuacán-Cuicatlán. Los climas predominantes son cálido seco y cálido semiseco, con una precipitación promedio de $495 \mathrm{~mm}$, con temperatura anual entre $16.3^{\circ} \mathrm{C}$ y $22{ }^{\circ} \mathrm{C}$, y precipitación media anual de $400 \mathrm{~mm}$. Las actividades productivas más importantes son la agricultura y la ganadería. La vegetación que predomina es el bosque tropical seco y bosque espinoso (García, 1981; INAFED, 2010). 
Muestreo en campo: En Pastorías se colocaron 11 cámaras-trampa digitales de la marca Cudde Back, de septiembre 2015 a septiembre 2016, la vegetación alrededor de estas estaciones de trampeo fueron fragmentos de bosque tropical seco, acahuales y pastizales. En Cuicatlán se establecieron diez cámarastrampa de la marca Primos, de octubre 2013 a julio 2014, y se ubicaron principalmente dentro del bosque tropical seco. Se abarcó dos temporadas climáticas contrastantes, lluvia y sequía. En Pastorías ocurrieron de junio a octubre y de noviembre a mayo respectivamente, mientras que para Cuicatlán fueron de junio a septiembre y de octubre a mayo. Las cámaras-trampa fueron colocadas en áreas donde se encontraron rastros como huellas, excretas o senderos, con una separación entre cada estación de trampeo mínima de $500 \mathrm{~m}$ lineales. Fueron programadas para registrar tres fotografías cada diez segundos.

Riqueza y diversidad de especies: LoS mamíferos medianos y grandes fotografiados se identificaron con base en literatura especializada (Reid, 1997; González-Christen, 2010; Aranda, 2012), para la nomenclatura taxonómica se siguió el trabajo de Ramírez-Pulido, González-Ruiz, Gardner, \& Arroyo-Cabrales (2014). Se determinó la riqueza específica durante todo el periodo de muestreo y para las temporadas de lluvia y de sequía. La diversidad verdadera se obtuvo a través de los números de la serie Hill (Hill, 1973) con el paquete vegan (Oksanen et al., 2017), y se comparó entre temporada del año y entre localidades mediante una prueba t-Student, con un valor de significancia de $\mathrm{P}<0.05$. El esfuerzo de muestreo se calculó sumando todos los días en que cada cámara-trampa permaneció activa. La diversidad verdadera al igual que el esfuerzo de muestreo se determinó para cada una de las cámaras-trampa debido a que la distribución de los registros fotográficos de las especies no es homogénea entre éstas. Se representaron las curvas de acumulación de riqueza de especies para cada localidad usando el número de especies por cámara-trampa mediante el paquete
BiodiversityR (Kindt \& Coe, 2005). Los paquetes de análisis se trabajaron en el programa estadístico R 3.4.0 (R Core Team, 2017).

Índices de abundancia relativa: Se estimaron los índices de abundancia relativa (IAR) para cada especie y por cada una de las cámarastrampa (Goulart et al., 2009). El IAR se obtuvo a través del conteo de registros independientes diferenciados por ciclos de 24 horas. Se consideró como registro independiente a las fotografías consecutivas de individuos de diferentes especies y a las fotografías de individuos de la misma especie separadas por más de 24 horas. En las fotografías donde aparecieron varios individuos de la misma especie o diferentes especies, los registros fueron considerados como el total de individuos capturados (Monroy-Vilchis, Zarco-González, Rodríguez-Soto, Soria-Díaz, \& Urios, 2011). El IAR se estimó para la $i$-especie, en la $j$-cámara durante la $k$-temporada del año (lluvia y sequía) para cada localidad (Pastorías y Cuicatlán), empleando la siguiente fórmula: $I A R_{i j k}=\mathrm{n} /$ días * 100; donde: $n=$ número de capturas o eventos fotográficos independientes, días $=$ esfuerzo de muestreo (número de cámaras-trampa por días de monitoreo) y $100=$ factor de corrección estándar. En consecuencia, el IAR se interpreta como el número de fotos promedio por cada 100 días. Se compararon los IAR de todo el conjunto de especies en cada localidad mediante un análisis de varianza de una vía (ANOVA), y una prueba a posteriori Tukey. Todos los análisis fueron hechos en el programa estadístico R 3.4.0 (R Core Team, 2017).

Relación con variables humanas y de hábitat: Con el fin de establecer la relación entre el número de fotos independientes y las covariables de hábitat y humanas, se aplicaron modelos lineales generalizados (MLG) tipo Quasipoisson (al ser la variable dependiente un dato de conteo), en los que se incluyó el esfuerzo de muestreo como una variable de corrección, debido a que el esfuerzo no fue igual entre temporadas (Crawley, 2013). Este análisis sólo se hizo para las especies con 
mayor número de registros. Todos los análisis se realizaron en el programa estadístico R 3.4.0 (R Core Team, 2017).

Se consideró como covariables de hábitat a los tipos de vegetación y las temporadas climáticas (lluvia/sequía). Se creó un buffer de 1 ha de extensión alrededor de cada cámara-trampa para tener representado el mosaico de vegetación y uso de suelo circundante, y el área que cubren $\left(\mathrm{m}^{2}\right)$; esto se obtuvo de una capa digital de uso de suelo y vegetación (INEGI, 2013b). Los tipos de vegetación y uso de suelo fueron: vegetación secundaria arbustiva de bosque tropical seco, vegetación secundaria arbórea de bosque tropical seco, pastizal y áreas de agricultura en Pastorías; bosque tropical seco, vegetación secundaria arbustiva de bosque tropical seco, vegetación secundaria arbórea de bosque tropical seco y áreas de agricultura en Cuicatlán. Las covariables de presencia humana alrededor de las cámaras-trampa se obtuvieron calculando la distancia al camino, carretera y localidad humana más cercana (m) (INEGI, 2013a). Esto se realizó a través del software ArcMap 10.0 (ESRI http://www.esri.com).

\section{RESULTADOS}

Riqueza y diversidad de especies: En Pastorías se registraron cuatro órdenes, 11 familias y 13 géneros de mamíferos medianos y grandes, con un esfuerzo de muestreo total de 3526 días/trampa (1411 en lluvia y 2115 en sequía); mientras que en Cuicatlán se identificaron cuatro órdenes, ocho familias y 13 géneros, el esfuerzo de muestreo fue de 2690 días/trampa (1218 en lluvia y 1472 en sequía) (Cuadro 1). La riqueza específica para ambas localidades fue de 13 especies. En Pastorías se registró una riqueza promedio por cámaratrampa de $3.55( \pm 2.42)$ especies, durante la temporada de sequía ésta fue mayor $(5.27 \pm$ 1.95) en comparación con la temporada de lluvia $(3.54 \pm 2.42)$. En Cuicatlán el registro fue de 3.70 ( \pm 2.91$)$ especies por cámara-trampa, mientras que en las temporadas el promedio de especies detectadas fue parecido (sequía $=3.60$ \pm 2.45 ; lluvia $=3.70 \pm 2.90$ ). En la primera localidad la diversidad verdadera obtuvo un valor de $3.69( \pm 0.80)$, y en la temporada de sequía fue mayor que la estimada en lluvias ( $3.40 \pm 0.85$ y $2.77 \pm 1.14$ respectivamente). En Cuicatlán la diversidad fue de $2.77( \pm 1.12)$, y durante las temporadas se obtuvieron valores similares (sequía $=2.65 \pm 1.05 ;$ lluvia $=2.63$ $\pm 1.01)$. Se encontraron diferencias estadísticamente significativas entre la diversidad verdadera de las dos localidades $(\mathrm{t}=2.12, \mathrm{P}=0.04)$, pero ocurrió lo contrario entre temporadas en ambas localidades. En Pastorías se observó una tendencia asintótica en la curva de acumulación de riqueza de especies, lo cual no se obtuvo en Cuicatlán (Fig. 1).

En Pastorías O. virginianus fue la especie registrada en más cámaras-trampa (diez de once), seguido de Canis latrans y U. cinereoargenteus (nueve y ocho cámaras). En Cuicatlán $O$. virginianus fue quien apareció en más cámaras (nueve), seguido de $U$. cinereoargenteus (cinco cámaras) (Cuadro 1). Cinco especies de las registradas en las dos localidades se encuentran bajo alguna categoría de riesgo de acuerdo con su estado de conservación dentro de la Norma Oficial Mexicana, NOM-059-2010 (SEMARNAT, 2010), estas fueron Tamandua mexicana, Herpailurus yagouaroundi, Leopardus wiedii, Lynx rufus y Galictis vittata (Cuadro 1).

Índice de abundancia relativa: Durante el periodo de estudio las especies con mayor abundancia relativa en las dos localidades fueron $U$. cinereoargenteus (Pastorías $3.90 \pm$ 7.16; Cuicatlán $3.52 \pm 7.50)$, O. virginianus (Pastorías $2.69 \pm 2.47$; Cuicatlán $4.18 \pm 3.81$ ) y C. latrans (Pastorías 2.16 \pm 5.13 ; Cuicatlán $3.17 \pm 8.52$ ) (Cuadro 1 y Fig. 2). En Pastorías la abundancia relativa de $U$. cinereoargentus fue estadísticamente diferente a la de nueve especies, pero no respecto a $O$. virginianus, $C$. latrans y Dhidelphis sp. En Cuicatlán, la abundancia relativa estimada para $O$. virginianus no fue estadísticamente diferente a la estimada para U. cinereoargentus, C. latrans, C. leuconotus y Dhidelphis sp., pero sí lo fue para las ocho especies restantes. 
CUADRO 1

Mamíferos medianos y grandes registrados en dos bosques tropicales secos del centro de México durante dos temporadas climáticas

TABLE 1

Medium and large mammals recorded in two tropical dry forests of central Mexico during two climatic seasons

\begin{tabular}{|c|c|c|c|c|c|c|c|c|c|}
\hline \multirow{2}{*}{ ORDEN/Familia/Especie } & \multirow{2}{*}{ Sitio } & \multirow{2}{*}{ CTR } & \multicolumn{2}{|c|}{ Periodo de estudio } & \multicolumn{2}{|r|}{ Lluvias } & \multicolumn{2}{|r|}{ Secas } & \multirow{2}{*}{ NOM-059 } \\
\hline & & & $\mathrm{n}$ & IAR & $\mathrm{n}$ & IAR & $\mathrm{n}$ & IAR & \\
\hline $\begin{array}{l}\text { DIDELPHIMORPHIA } \\
\text { Didelphidae }\end{array}$ & & & & & & & & & \\
\hline Didelphis sp. & Pas & 3 & 27 & $1.03 \pm 3.90$ & 1 & $0.06 \pm 0.2$ & 26 & $1.99 \pm 5.5$ & \\
\hline $\begin{array}{l}\text { Didelphis sp. } \\
\text { CINGULATA } \\
\text { Dasypodinae }\end{array}$ & Cui & 1 & 15 & $0.50 \pm 2.08$ & 1 & $0.08 \pm 0.2$ & 14 & $0.93 \pm 2.9$ & \\
\hline $\begin{array}{l}\text { Dasypus novemcinctus } \\
\text { PILOSA } \\
\text { Myrmecophagidae }\end{array}$ & Pas & 5 & 27 & $0.71 \pm 1.75$ & 14 & $0.87 \pm 2.3$ & 13 & $0.55 \pm 1.1$ & \\
\hline $\begin{array}{l}\text { Tamandua mexicana } \\
\text { LAGOMORPHA } \\
\text { Leporidae }\end{array}$ & Pas & 2 & 3 & $0.07 \pm 0.22$ & 0 & 0 & 3 & $0.13 \pm 0.3$ & $\mathrm{P}$ \\
\hline $\begin{array}{l}\text { Sylvilagus floridanus } \\
\text { CARNIVORA } \\
\text { Felidae }\end{array}$ & Cui & 1 & 8 & $0.31 \pm 1.37$ & 8 & $0.61 \pm 1.9$ & 0 & 0 & \\
\hline Herpailurus yagouaroundi & Pas & 1 & 1 & $0.03 \pm 0.15$ & 1 & $0.06 \pm 0.2$ & 0 & 0 & A \\
\hline Leopardus wiedii & $\begin{array}{l}\text { Pas } \\
\text { Cui }\end{array}$ & $\begin{array}{l}2 \\
1\end{array}$ & $\begin{array}{l}2 \\
1\end{array}$ & $\begin{array}{l}0.07 \pm 0.25 \\
0.03 \pm 0.15\end{array}$ & $\begin{array}{l}0 \\
0\end{array}$ & $\begin{array}{l}0 \\
0\end{array}$ & $\begin{array}{l}2 \\
1\end{array}$ & $\begin{array}{l}0.14 \pm 0.3 \\
0.07 \pm 0.2\end{array}$ & $\mathrm{P}$ \\
\hline $\begin{array}{l}\text { Lynx rufus } \\
\text { Canidae }\end{array}$ & Cui & 3 & 12 & $0.42 \pm 0.93$ & 4 & $0.31 \pm 1.0$ & 8 & $0.53 \pm 0.9$ & $\mathrm{P}$ \\
\hline Canis latrans & $\begin{array}{l}\text { Pas } \\
\text { Cui }\end{array}$ & $\begin{array}{l}9 \\
3\end{array}$ & $\begin{array}{l}79 \\
87\end{array}$ & $\begin{array}{l}2.16 \pm 5.13 \\
3.17 \pm 8.52\end{array}$ & $\begin{array}{l}44 \\
49\end{array}$ & $\begin{array}{c}2.77 \pm 7.1 \\
3.74 \pm 10.5\end{array}$ & $\begin{array}{l}35 \\
38\end{array}$ & $\begin{array}{l}1.55 \pm 2.0 \\
2.59 \pm 6.4\end{array}$ & \\
\hline Urocyon cinereoargenteus & $\begin{array}{l}\text { Pas } \\
\text { Cui }\end{array}$ & $\begin{array}{l}8 \\
5\end{array}$ & $\begin{array}{c}133 \\
97\end{array}$ & $\begin{array}{l}3.90 \pm 7.16 \\
3.52 \pm 7.50\end{array}$ & $\begin{array}{l}11 \\
52\end{array}$ & $\begin{array}{l}0.90 \pm 1.4 \\
3.97 \pm 8.7\end{array}$ & $\begin{array}{c}122 \\
45\end{array}$ & $\begin{array}{l}6.91 \pm 9.3 \\
3.06 \pm 6.6\end{array}$ & \\
\hline Mephitidae & & & & & & & & & \\
\hline Conepatus leuconotus & $\begin{array}{l}\text { Pas } \\
\text { Cui }\end{array}$ & $\begin{array}{l}7 \\
1\end{array}$ & $\begin{array}{l}18 \\
22\end{array}$ & $\begin{array}{l}0.52 \pm 1.04 \\
0.83 \pm 3.41\end{array}$ & $\begin{array}{l}11 \\
20\end{array}$ & $\begin{array}{l}0.68 \pm 1.4 \\
1.53 \pm 4.8\end{array}$ & $\begin{array}{l}7 \\
2\end{array}$ & $\begin{array}{l}0.35 \pm 0.5 \\
0.13 \pm 0.4\end{array}$ & \\
\hline Mephitis macroura & $\begin{array}{l}\text { Pas } \\
\text { Cui }\end{array}$ & $\begin{array}{l}1 \\
1\end{array}$ & $\begin{array}{l}1 \\
1\end{array}$ & $\begin{array}{l}0.02 \pm 0.11 \\
0.04 \pm 0.17\end{array}$ & $\begin{array}{l}0 \\
1\end{array}$ & $\begin{array}{c}0 \\
0.08 \pm 0.2\end{array}$ & $\begin{array}{l}1 \\
0\end{array}$ & $\begin{array}{c}0.05 \pm 0.1 \\
0\end{array}$ & \\
\hline Mustelidae & & & & & & & & & \\
\hline $\begin{array}{l}\text { Galictis vittata } \\
\text { Procyonidae }\end{array}$ & Pas & 1 & 1 & $0.03 \pm 0.15$ & 1 & $0.06 \pm 0.2$ & 0 & 0 & A \\
\hline Bassariscus astutus & Cui & 2 & 9 & $0.32 \pm 0.72$ & 5 & $0.38 \pm 0.8$ & 4 & $0.27 \pm 0.6$ & \\
\hline Nasua narica & $\begin{array}{l}\text { Pas } \\
\text { Cui }\end{array}$ & $\begin{array}{l}5 \\
4\end{array}$ & $\begin{array}{c}13 \\
6\end{array}$ & $\begin{array}{l}0.42 \pm 1.25 \\
0.22 \pm 0.43\end{array}$ & $\begin{array}{l}3 \\
5\end{array}$ & $\begin{array}{l}0.19 \pm 0.4 \\
0.38 \pm 0.5\end{array}$ & $\begin{array}{c}10 \\
1\end{array}$ & $\begin{array}{l}0.65 \pm 1.7 \\
0.07 \pm 0.2\end{array}$ & \\
\hline Procyon lotor & $\begin{array}{l}\text { Pas } \\
\text { Cui }\end{array}$ & $\begin{array}{l}3 \\
1\end{array}$ & $\begin{array}{c}37 \\
1\end{array}$ & $\begin{array}{l}0.95 \pm 2.31 \\
0.04 \pm 0.17\end{array}$ & $\begin{array}{l}8 \\
1\end{array}$ & $\begin{array}{l}0.50 \pm 1.6 \\
0.08 \pm 0.2\end{array}$ & $\begin{array}{c}29 \\
0\end{array}$ & $\begin{array}{c}1.40 \pm 2.8 \\
0\end{array}$ & \\
\hline $\begin{array}{l}\text { ARTIODACTYLA } \\
\text { Tayassuidae }\end{array}$ & & & & & & & & & \\
\hline $\begin{array}{l}\text { Dicotyles angulatus } \\
\text { Cervidae }\end{array}$ & Cui & 2 & 5 & $0.22 \pm 0.72$ & 3 & $0.30 \pm 0.9$ & 2 & $0.13 \pm 0.4$ & \\
\hline Odocoileus virginianus & $\begin{array}{l}\text { Pas } \\
\text { Cui }\end{array}$ & $\begin{array}{c}10 \\
9\end{array}$ & $\begin{array}{l}100 \\
114\end{array}$ & $\begin{array}{l}2.69 \pm 2.47 \\
4.18 \pm 3.81\end{array}$ & $\begin{array}{l}29 \\
57\end{array}$ & $\begin{array}{l}2.02 \pm 1.8 \\
4.52 \pm 2.9\end{array}$ & $\begin{array}{l}71 \\
57\end{array}$ & $\begin{array}{l}3.36 \pm 2.9 \\
3.85 \pm 4.7\end{array}$ & \\
\hline
\end{tabular}

Pas $=$ Pastorías; Cui $=$ Cuicatlán $; \mathrm{CTR}=$ Cámaras-trampa con registros; $\mathrm{n}=$ Número de registros independientes; $\mathrm{IAR}=$ Índices de Abundancia Relativa (promedio \pm desviación estándar); NOM-059-SEMARNAT, A = Amenazada, $\mathrm{P}=$ En peligro de extinción. 

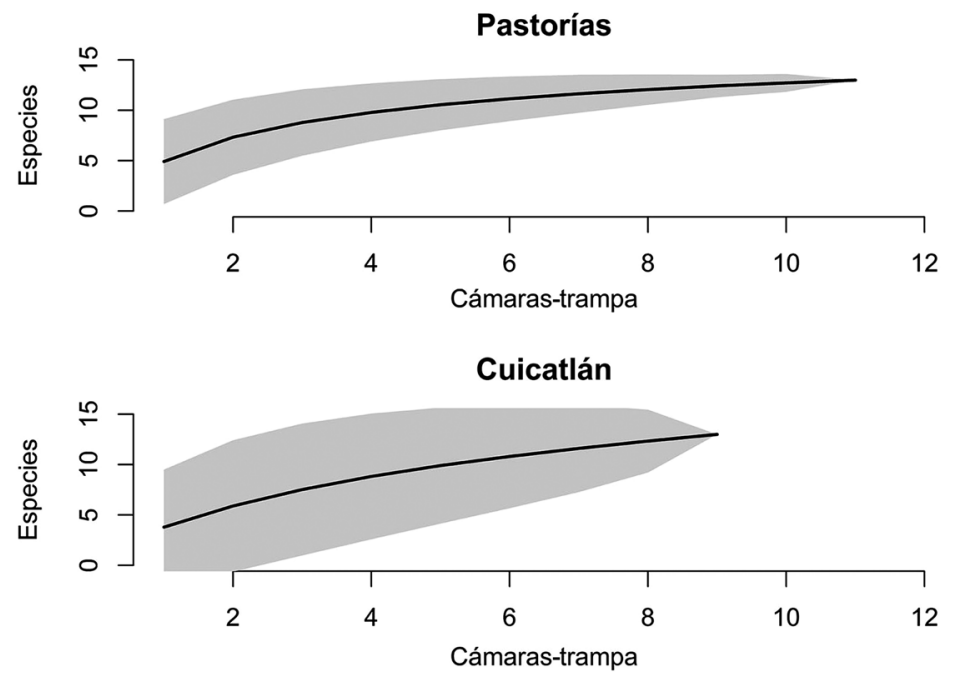

Fig. 1. Curva de acumulación de especies de mamíferos medianos y grandes basadas en las unidades de muestreo (cámaratrampa) en las dos localidades de estudio.

Fig. 1. Species accumulation curve of medium and large mammals based on sampling units (camera-trap) in the two study sites.
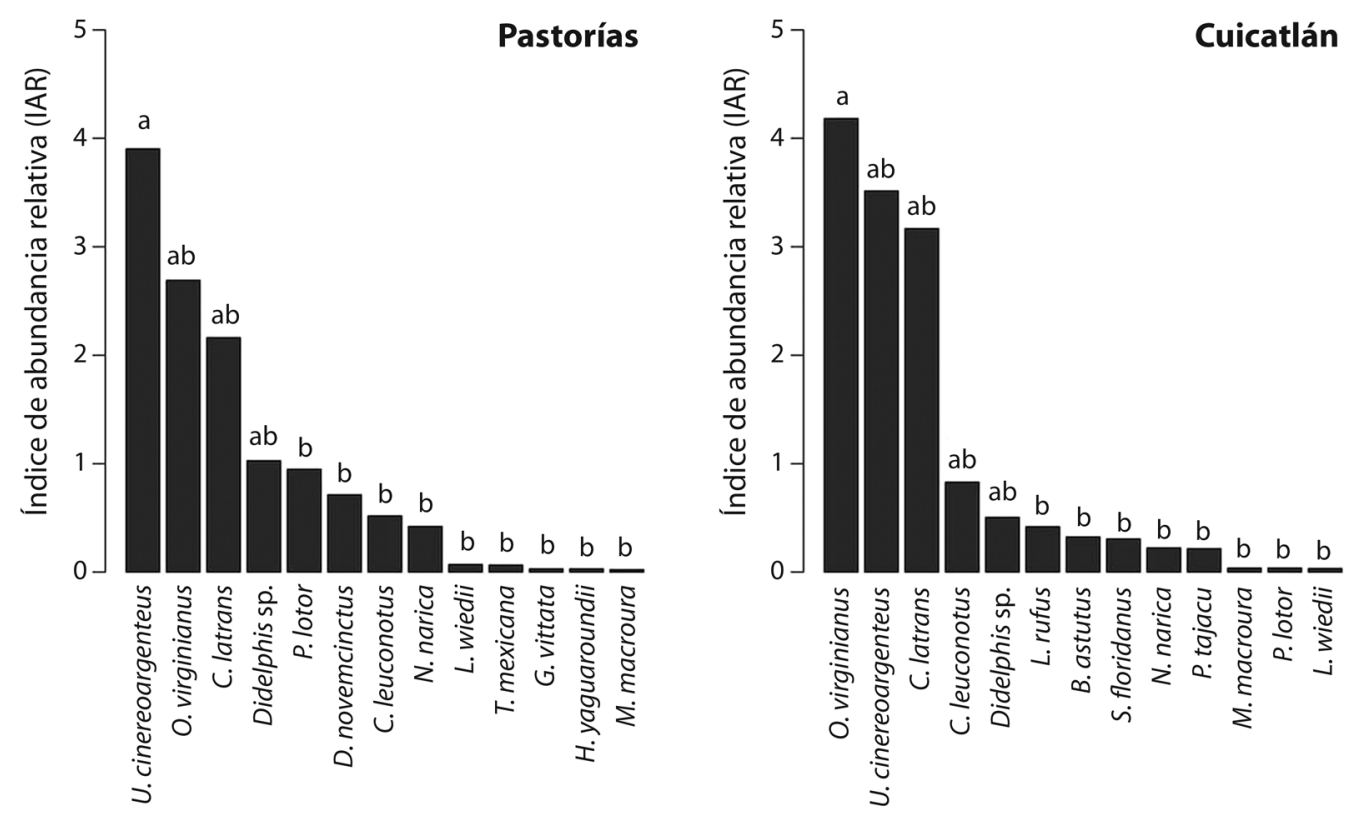

Fig. 2. Índice de abundancia relativa (IAR) de los mamíferos medianos y grandes de las dos localidades de estudio. Las letras encima de las barras indican el resultado de la prueba a posteriori Tukey, letras similares indican no diferencia.

Fig. 2. Relative abundance index (IAR) of the medium and large mammals of the two study sites. The letters above the bars indicate the result of the a posteriori Tukey test, similar letters indicate no difference. 
CUADRO 2

Variables que influyen en el registro de los mamíferos medianos y grandes a través del foto-trampeo en dos bosques tropicales secos del centro de México

TABLE 2

Variables that influence the registration of medium and large mammals through photo-trapping in two tropical dry forests of central Mexico

\begin{tabular}{lcc}
\multicolumn{1}{c}{ Especie } & Sitio & Modelo \\
Canis latrans & Pas & $-3.35^{*}+-1.01$ TS \\
Urocyon cinereoargenteus & Pas & $-6.13^{*}+1.41 \mathrm{TS}+0.53^{*}$ VSarb $+-4.21 \mathrm{Ag}$ \\
& Cui & $-3.03^{*}+-0.45 \mathrm{TS}$ \\
Odocoileus virginianus & Pas & $-3.89^{*}+0.28 \mathrm{TS}+1.0 .55^{*} \mathrm{DL}$ \\
& Cui & $-3.05^{*}+-0.15 \mathrm{TS}$ \\
\hline
\end{tabular}

Pas = Pastorías, Cui $=$ Cuicatlán; $T S=$ Temporada seca $D L=$ Distancia a localidad VSarb = Vegetación secundaria arbórea; $A g=$ Áreas de agricultura; ${ }^{*}=\mathrm{P}<0.05$.

Relación con variables humanas y de hábitat: Los MLG se hicieron para U. cinereoargenteus, $O$. virginianus y $C$. latrans; en Cuicatlán no fue posible realizar el MLG de esta última especie debido al agrupamiento de registros en una sola cámara. De acuerdo con los MLG la temporada de lluvias fue la variable estadísticamente significativa $(\mathrm{P}<0.05)$ que influyó de forma negativa en el registro de las tres especies en ambas localidades (Cuadro 2), mientras que en Pastorías la detección de $U$. cinereoargenteus y $O$. virginianus también estuvo influenciada por variables que se relacionan con la presencia humana $(\mathrm{P}<0.05)$ (Cuadro 2).

\section{DISCUSIÓN}

Los resultados de este estudio reflejan que a pesar de la presión antrópica en Pastorías, los relictos de bosque tropical seco aún mantienen una alta riqueza de especies, similar a la de Cuicatlán que es un sitio conservado. En ambas localidades de estudio se registró el mismo número de especies, pero con una composición diferente. La riqueza promedio por cámaratrampa durante todo el periodo de estudio también fue muy parecida entre localidades, esto a pesar de que la curva de acumulación de especies sólo alcanzó una tendencia asintótica en la localidad de Pastorías. Es posible que esto sea efecto del tamaño del área de estudio, al ser Cuicatlán un sitio más grande no fue posible detectar a todas las especies. En las áreas naturales protegidas puede encontrarse una alta riqueza y diversidad de especies de fauna, debido principalmente a la protección que estos sitios les confieren respecto a la cacería y conservación de su hábitat (Bruner, Gullison, Rice, \& Da Fonseca, 2001). Sin embargo, cerca de la quinta parte del total de las especies de mamíferos en México habitan fuera de las ANP (Vázquez \& Valenzuela-Galván, 2009), por lo que es necesario enfocar también la atención hacia aquellas zonas ricas en biodiversidad, pero altamente vulnerables de desaparecer.

Los mamíferos medianos y grandes registrados en Cuicatlán ya han sido previamente reportados en otras localidades al interior de la RBTC (Briones-Salas, 2000; Cruz-Jácome et al., 2015). La presencia de dos especies de felinos (Leopardus wiedii y Lynx rufus) considerados en peligro de extinción para México (SEMARNAT, 2010) resalta la importancia de esta localidad para la conservación de mamíferos dentro de la RBTC. De las especies de mamíferos medianos y grandes que se sabe habitan al interior de esta reserva (Botello et al., 2006a, 2006b, 2013; Cruz-Jácome et al., 2015), este estudio no registró nutria (Lontra longicaudis), armadillo (Dasypus novemcinctus), zorrillo manchado (Spilogale 
angustifrons) y dos especies de felinos (Puma concolor y Herpailurus yagouaroundi), tampoco fue posible identificar a cuál especie de tlacuache pertenecían los registros que se obtuvieron (Didelphis marsupialis o D. virginiana) debido a la calidad de las fotos. En cuanto a L. longicaudis es importante considerar que el muestreo de campo no fue dirigido hacia especies con hábitos acuáticos, y en el caso de los dos felinos es posible que su comportamiento evasivo y la mayor área de uso disponible dentro de la reserva hayan tenido efecto en su detección. Por otra parte, la riqueza específica de esta localidad es similar a la reportada en otros bosques tropicales secos en el estado de Oaxaca y fuera de la RBTC, donde los valores van de 13 a 18 especies (Santos-Moreno \& Ruíz-Velásquez, 2011; Cervantes \& Riveros, 2012; Cortés-Marcial \& Briones-Salas, 2014), con base en esto, consideramos que se hizo un buen esfuerzo de muestreo, pero es necesario aumentar el número de días de trabajo si se busca registrar el total de especies de mamíferos medianos y grandes que habitan el sitio.

Este trabajo reporta por primera vez un listado de especies y aspectos poblacionales de los mamíferos medianos y grandes que habitan en la localidad de Pastorías, lo cual contribuye a ampliar la información con que se cuenta para el bosque tropical seco del estado de Veracruz. Las especies registradas en este trabajo han sido previamente reportadas para La Mancha y sus alrededores (Morales-Mávil \& Guzmán-Guzmán, 1994; González-Romero \& Lara-López, 2006; Medrano-Nájera, RamírezPinero, \& Guevara-Sada, 2014). El registro de cuatro especies (Leopardus wiedii, Herpailurus yaguaroundi, Tamandua mexicana y Galictis vittata) catalogadas bajo amenaza y peligro de extinción para México (SEMARNAT, 2010) denota la importancia de esta región en cuanto a la conservación de la fauna.

Por otra parte, la diversidad verdadera fue diferente entre las dos localidades, en promedio Pastorías tuvo un valor mayor que Cuicatlán, este resultado puede estar asociado a la composición heterogénea de Pastorías, donde la vegetación alrededor de las estaciones de foto-trampeo fueron el bosque tropical seco, acahuales, pastizales y zonas de agricultura, lo que favorece una mayor diversidad de alimento para los individuos. De acuerdo con lo que se observó en la variación de la diversidad registrada entre cámaras-trampa en Pastorías, las especies podrían estarse moviendo más para acceder a los recursos necesarios entre los parches de vegetación. Es importante considerar que, a pesar de que la diversidad es mayor en Pastorías, el número de registros fotográficos de las especies no es alto, lo que puede indicar que la calidad del hábitat no es adecuada para mantener números poblacionales grandes, lo que se reflejó también en los índices de abundancia relativa.

Para ambos sitios de estudio las especies más abundantes de acuerdo con el IAR fueron $U$. cinereoargenteus, $C$. latrans, y $O$. virginia$n u s$, esto coincide con lo reportado en otros estudios en bosque tropical seco y al interior de la RBTC (Monroy-Vilchis et al., 2011; Cruz-Jácome et al., 2015). Se sabe que U. cinereoargenteus, $C$. latrans, y $O$. virginianus son especies tolerantes a cierto grado de alteración del hábitat y que incluso pueden verse beneficiadas por cambios en el uso de suelo (HidalgoMihart, Cantú-Salazar, González-Romero, \& López-González, 2004; Galindo-Leal \& Weber, 2005; Servín \& Chacón, 2005). Los hábitos omnívoros de las dos primeras especies permiten hacer uso de diferentes ambientes, y con ello tener mayor ventaja sobre otros mamíferos al poder hacer uso de más recursos (Guerrero, Badii, Zalapa, \& Flores, 2002).

La temporada climática fue la variable que estuvo relacionada en todos los casos a la detección de $U$. cinereoargenteus, $C$. latrans, y $O$. virginianus, la relación negativa con la temporada de lluvias podría ser a causa de la menor detectabilidad que hay en esta temporada debido al aumento de la cobertura vegetal. Por otra parte, para $O$. virginianus se ha reportado que durante esta temporada aumentan sus movimientos (Bello, Gallina, \& Equihua, 2004), lo que puede ocasionar que los individuos se desplacen hacia sitios que no se cubrieron con el muestreo. 
Finalmente, el bosque tropical seco del centro de Veracruz es un lugar importante para la conservación de fauna silvestre, sin embargo, la abundancia relativa de muchas especies de mamíferos medianos y grandes reportadas en este estudio es baja, esto puede ser el reflejo del estado de conservación del sitio. En este sentido, es importante que las instancias de gobierno correspondientes generen planes de manejo adecuados para mantener la biodiversidad que persiste en estos ecosistemas, y que a su vez estos se integren con alternativas que permitan a las comunidades humanas que se encuentran en desarrollo, la convivencia con las poblaciones de fauna silvestre y el mantenimiento de su hábitat. La información recabada en este estudio es relevante para ampliar el conocimiento de las poblaciones de mamíferos medianos y grandes que habitan al interior de la RBTC, donde aún es necesario el desarrollo de más estudios para conocer otros parámetros poblacionales de las especies, con el fin de mejorar los planes de manejo de esta reserva.

\section{AGRADECIMIENTOS}

Este trabajo fue financiado mediante el proyecto No. 20035-30918 del Instituto de Ecología, A. C., el convenio No. 10558 con la Asociación de Unidades de Manejo de Veracruz (ASUMAVER) y la Secretaria de Medio Ambiente y Recursos Naturales (SEMARNAT), y el Proyecto CB-2009-01 No. 130702 del Consejo Nacional de Ciencia y Tecnología (CONACyT). Agradecemos al personal de la ASUMAVER y de la UMA Cruztepeque. A los pobladores y autoridades de San Juan Bautista, Cuicatlán, así como a la Comisión Nacional de Áreas Naturales Protegidas - Reserva de la Biosfera de Tehuacán-Cuicatlán. A todos los estudiantes que participaron durante los muestreos de campo dentro del proyecto "Evaluación de las interacciones entre el venado cola blanca y el ganado en la Reserva de Biosfera de Tehuacán-Cuicatlán: implicaciones de conservación y manejo". A Policarpo Ronzón-Pérez por su apoyo en campo, así como a Adriana Sandoval-Comte por su apoyo en el uso de
Sistemas de Información Geográfica, a Teresa Pérez-Pérez por su apoyo logístico.

\section{RESUMEN}

El bosque tropical seco es uno de los ecosistemas más amenazados alrededor del mundo, principalmente a causa de la deforestación. En México más de la mitad de su superficie ha desaparecido, lo que ha provocado pérdida de biodiversidad. El objetivo de este estudio fue comparar la riqueza, diversidad y abundancia relativa de la comunidad de mamíferos medianos y grandes en dos sitios con diferente manejo y estatus de conservación, y donde está presente el bosque tropical seco. También se buscó conocer las variables del hábitat y de influencia humana que se relacionan con la presencia de las especies más comunes. Los sitios de estudio fueron Pastorías, Actopan, Veracruz y San Juan Bautista Cuicatlán, Oaxaca, México. El primer sitio está muy transformado por las actividades antrópicas mientras que el segundo se encuentra dentro de la Reserva de la Biosfera de Tehuacán-Cuicatlán (RBTC). En Pastorías se establecieron 11 cámaras-trampa de septiembre 2015 a septiembre 2016; mientras que en Cuicatlán 10 cámaras-trampa de octubre 2013 a julio 2014. El esfuerzo de muestreo fue de 3526 días/trampa y 443 registros independientes, y 2690 días/trampa y 378 registros independientes para cada sitio respectivamente. En cada localidad se registraron 13 especies. Tres de estas especies están en peligro de extinción y dos bajo amenaza. Se encontraron diferencias estadísticamente significativas entre la diversidad verdadera de mamíferos medianos y grandes entre localidades. De acuerdo con el índice de abundancia relativa (IAR), las especies más abundantes fueron: Odocoileus virginianus, Urocyon cinereoargenteus y Canis latrans. Los análisis de regresión sugieren que las variables de temporada, la cobertura de vegetación secundaria arbórea y la distancia a la localidad más cercana se relacionan con el registro de estas especies. Los remanentes de bosque tropical seco en Pastorías mantienen una alta riqueza de especies, la cual es muy parecida a la de un sitio conservado como Cuicatlán. Sin embargo, el bajo número de registros fotográficos puede indicar que la calidad del hábitat no es adecuada. La información recabada en este estudio puede ser útil para el desarrollo de programas para conservar el bosque tropical seco del centro de Veracruz y contribuye con la ampliación del conocimiento que se tiene sobre la comunidad de mamíferos que habitan en la RBTC.

Palabras clave: foto-trampeo; riqueza de especies; abundancia relativa; Veracruz, Oaxaca.

\section{REFERENCIAS}

Ahumada, J. A., Silva, C. E., Gajapersad, K., Hallam, C., Hurtado, J., Martin, E., \& Sheil, D. (2011). Community structure and diversity of tropical forest 
mammals: data from a global camera trap network. Philosophical Transactions of the Royal Society B: Biological Sciences, 366(1578), 2703-2711.

Aranda, S. J. M. (2012). Manual para el rastreo de mamiferos silvestres de México. México: Comisión Nacional para el Conocimiento y Uso de la Biodiversidad (CONABIO).

Baillie, J. E., Collen, B., Amin, R., Akcakaya, H. R., Butchart, S. H., Brummitt, N., ... Mace, G. M. (2008). Toward monitoring global biodiversity. Conservation Letters, 1(1), 18-26.

Balvanera, P., Islas, A., Aguirre, E., \& Quijas, S. (2000). Las selvas secas. Ciencias, 57, 19-24.

Bello, J., Gallina, S., \& Equihua, M. (2004). Movements of the white-tailed deer and their relationship with precipitation in northeastern Mexico. Interciencia, 29(7), 357-361.

Botello, F., Illoldi-Rangel, P., Linaje, M., \& SánchezCordero, V. (2006a). Primer registro del tigrillo (Leopardus wiedii, Schinz 1821) y del gato montés (Lynx rufus, Kerr 1792) en la Reserva de la Biosfera de Tehuacán-Cuicatlán, Oaxaca, México. Acta Zoológica Mexicana, 22(1), 135-139.

Botello, F., Salazar, J. M., Illoldi-Rangel, P., Linaje, M., Monroy, G., Duque, D., \& Sánchez-Cordero, V. (2006b). Primer registro de la nutria neotropical de río (Lontra longicaudis) en la Reserva de la Biosfera de Tehuacán-Cuicatlán, Oaxaca, México. Revista Mexicana de Biodiversidad, 77(1), 133-135.

Botello, F., Villaseñor, E., Guevara, L., Méndez, Á., Cortés, A., Iglesias, J., ... Salazar, J. M. (2013). Registros notables del zorrillo manchado (Spilogale angustifrons) y del jaguarundi (Puma yagouaroundi) en la Reserva de la Biosfera de Tehuacán-Cuicatlán, Oaxaca, México. Revista Mexicana de Biodiversidad 84(2), 713-717.

Briones-Salas, M. (2000). Lista anotada de los mamíferos de la región de La Cañada, en el valle de TehuacánCuicatlán, Oaxaca, México. Acta Zoológica Mexicana, 81, 83-103.

Bruner, A. G., Gullison, R. E., Rice, R. E., \& Da Fonseca, G. A. (2001). Effectiveness of parks in protecting tropical biodiversity. Science, 291(5501), 125-128.

Castillo-Campos, G., Avendaño, S., \& Medina, M. E. (2011). Flora y vegetación. En Comisión Nacional para el Conocimiento y Uso de la Biodiversidad (CONABIO) (Eds.), La biodiversidad en Veracruz: Estudio de Estado (pp. 163-179). México: CONABIO, Gobierno del Estado de Veracruz, Universidad Veracruzana, Instituto de Ecología, A.C.

Ceballos, G., \& Garcia, A. (1995). Conserving neotropical biodiversity: the role of dry forests in western Mexico. Conservation Biology, 9(6), 1349-1353.
Ceballos, G. \& Valenzuela, D. (2010). Diversidad, ecología y conservación de los vertebrados de Latinoamérica. En G. Ceballos, L. Martínez, A. García, E. Espinoza, J. B., Creel, \& R. Dirzo (Eds.), Diversidad, amenazas y áreas prioritarias para la conservación de las selvas secas del Pacífico de México (pp. 93-118). México: Fondo de Cultura Económica, Comisión Nacional para el Conocimiento y Uso de la Biodiversidad (CONABIO), Comisión Nacional de Áreas Naturales Protegidas (CONANP), WWF-México, Ecociencia S. C. \& TELMEX

Cervantes, F. A., \& Riveros, B. (2012). Mamíferos del Municipio de Cosoltepec, Oaxaca, México. Therya, 3(3), 311-325.

CONANP (Comisión Nacional de Áreas Naturales Protegidas). (2004). Reserva de la Biosfera TehuacánCuicatlán. México: CONANP. Recuperado de http:// www.conanp.gob.mx/anp/tehuacán-cuicatlán/

Cortés-Marcial, M. \& Briones-Salas, M. (2014). Diversidad, abundancia relativa y patrones de actividad de mamíferos medianos y grandes en una selva seca del Istmo de Tehuantepec, Oaxaca, México. Revista de Biología Tropical, 62(4), 1433-1448.

Crawley, M. J. (2013). The R Book (2nd Ed.). England: John Wiley \& Sons West Sussex.

Cruz-Jácome, O., López-Tello, E., Delfín-Alfonso, C. A., \& Mandujano, S. (2015). Riqueza y abundancia relativa de mamíferos medianos y grandes en una localidad en la Reserva de la Biosfera Tehuacán-Cuicatlán, Oaxaca, México. Therya, 6(2), 435-448.

Dávila, P., Arizmendi, M., Valiente-Banuet, A., Villaseñor, J. L., Casas, A., \& Lira, R. (2002). Biological diversity in the Tehuacán-Cuicatlán Valley, Mexico. Biodiversity and Conservation, 11(3), 421-442.

Dirzo, R. \& Ceballos, G. (2010). Las selvas secas de México: un reservorio de biodiversidad y laboratorio viviente. En G. Ceballos, L. Martínez, A. García, E. Espinoza, J. B. Creel, \& R. Dirzo (Eds.), Diversidad, amenazas y áreas prioritarias para la conservación de las selvas secas del Pacífico de México (pp. 13-17). México: Fondo de Cultura Económica, Comisión Nacional para el Conocimiento y Uso de la Biodiversidad (CONABIO), Comisión Nacional de Áreas Naturales Protegidas (CONANP), WWFMéxico, Ecociencia S. C. \& TELMEX.

Galindo-Leal, C. \& Weber, M. (2005). Venado cola blanca (Odocoileus virginianus Zimmermann, 1780). En G. Ceballos \& G. Oliva (Eds.), Los mamíferos silvestres de México (pp. 517-521). México: Comisión Nacional para el Conocimiento y Uso de la Biodiversidad (CONABIO) y Fondo de la Cultura Económica.

García, E. (1988). Modificaciones al sistema de clasificación climática de Koppen. México: Instituto de Geografía, Universidad Nacional Autónoma de México (UNAM) 
González-Christen, A. (2010). Los mamíferos de Veracruz: distribución, endemismo y estado de conservación. México, D.F.: Comisión Nacional para el Conocimiento y Uso de la Biodiversidad (CONABIO), Gobierno del Estado de Veracruz, Universidad Veracruzana, Instituto de Ecología, A.C.

González-Romero, A. \& Lara-López, M. (2006). Los anfibios, reptiles y mamíferos. In P. Moreno-Casasola (Ed.). Entornos veracruzanos: la costa de La Mancha (pp. 407-422). México: Instituto de Ecología, A. C.

Goulart, F. V. B., Cáceres, N. C., Graipel, M. E., Tortato, M. A., Ghizoni, I. R., \& Oliveira-Santos, L. G. R. (2009). Habitat selection by large mammals in a southern Brazilian Atlantic Forest. Mammalian Biology-Zeitschrift für Säugetierkunde, 74(3), 182-190.

Guerrero, S., Badii, M. H., Zalapa, S. S., \& Flores, A. E. (2002). Dieta y nicho de alimentación del coyote, zorra gris, mapache y jaguarundi en un bosque tropical caducifolio de la costa sur del estado de Jalisco, México. Acta Zoológica Mexicana, 86, 119-137.

Hidalgo-Mihart, M. G., Cantú-Salazar, L., GonzálezRomero, A., \& López-González, C. A. (2004). Historical and present distribution of coyote (Canis latrans) in Mexico and Central America. Journal of Biogeography, 31(12), 2025-2038.

Hill, M. O. (1973). Diversity and evenness: a unifying notation and its consequences. Ecology, 54(2), 427-432.

INAFED (Instituto Nacional para el Federalismo y el Desarrollo Municipal). (2010). Enciclopedia de los Municipios y delegaciones de México. México: INAFED. Recuperado de http://www.inafed.gob.mx/work/enciclopedia/EMM30veracruz/municipios/30004a.html

INEGI (Instituto Nacional de Estadística y Geografía). (2009). Prontuario de información geográfica municipal de los Estados Unidos Mexicanos. México: INEGI. Recuperado de http://www3.inegi.org.mx/ sistemas/mexicocifras/datos-geograficos/30/30004. pdf

INEGI (Instituto Nacional de Estadística y Geografía). (2013a). Conjunto de datos vectoriales de información topográfica escala 1:50 000 serie III. México: INEGI.

INEGI (Instituto Nacional de Estadística y Geografía) (2013b). Uso de suelo y vegetación, escala 1:250000, serie V (capa unión), escala 1:250000. México: INEGI.

Kindt, R., \& Coe, R. (2005). Tree diversity analysis. A manual and software for common statistical methods for ecological and biodiversity studies. Nairobi: World Agroforestry Centre (ICRAF).

Medrano-Nájera, R., Ramírez-Pinero, M., \& GuevaraSada, S. (2014). Una mirada a la dispersión de semillas en las excretas de mamíferos. Cuadernos de Biodiversidad, 46, 19-28.

Miles, L., Newton, A. C., DeFries, R. S., Ravilious, C., May, I., Blyth, S., \& Gordon, J. E. (2006). A global overview of the conservation status of tropical dry forests. Journal of Biogeography, 33(3), 491-505.

Monroy-Vilchis, O., Zarco-González, M. M., RodríguezSoto, C., Soria-Díaz, L., \& Urios, V. (2011). Fototrampeo de mamíferos en la Sierra Nanchititla, México: abundancia relativa y patrón de actividad. Revista de Biología Tropical, 59(1), 373-383.

Morales-Mávil, J. E., \& Guzmán-Guzmán, S. (1994). Fauna silvestre de la zona de La Mancha, Veracruz, México. La ciencia y el Hombre, 16, 77-103.

Moreno, C. E. (2001). Métodos para medir la biodiversidad (Vol. 1.). Zaragoza, España: M\&T-Manuales y Tesis SEA.

Moreno-Casasola, P. (2006). Entornos veracruzanos: la costa de La Mancha. México: Instituto de Ecología, A. C.

Oksanen, J., Blanchet, F. G., Friendly, M., Kindt, R., Legendre, P., McGlinn, D., ... Wagner, H. (2017). Vegan: Community Ecology Package. Retrieved from https:/CRAN.R-project.org/package=vegan

Ortíz-García, A. I., Ramos-Robles, M. I., Pérez-Solano, L. A., \& Mandujano, S. (2012). Distribución potencial de los ungulados silvestres en la Reserva de Biosfera de Tehuacán-Cuicatlán, México. Therya, 3(3), 334-348.

R Core Team (2017). R: A language and environment for statistical computing. R Foundation for Statistical Computing, Vienna, Austria. Retrieved from https:// www.R-project.org/

Ramírez-Pulido, J., González-Ruiz, N., Gardner, A. L., \& Arroyo-Cabrales, J. (2014). List of recent land mammals of Mexico, 2014. Special Publications Museum of Texas Tech University, 63, 1-69.

Reid, F. (1997). A field guide to the mammals of Central America and Southeast Mexico. Oxford, United Kingdom: Oxford University Press.

Rzedowski, J. 1978. Vegetación de México. México, D.F.: Limusa

Santos-Moreno, A., \& Ruiz-Velásquez, E. (2011). Diversidad de mamíferos de la región de Nizanda, Juchitán, Oaxaca, México. Therya, 2(2), 155-168.

SEMARNAT (Secretaría de Medio Ambiente y Recursos Naturales). (2010). Norma Oficial Mexicana NOM-059-SEMARNAT-2010. Protección ambiental, 
especies nativas de flora y fauna silvestres de México, categorías de riesgo y especificaciones para su inclusión, exclusión o cambio, y lista de especies en riesgo. México: Diario Oficial de la Federación.

Servín, J., \& Chacón, E. 2005. Zorra gris (Urocyon cinereoargenteus Schreber, 1775). En G. Ceballos \& G Oliva (Eds.), Los mamíferos silvestres de México (pp. 354-355). México: Comisión Nacional para el Conocimiento y Uso de la Biodiversidad (CONABIO) y Fondo de la Cultura Económica.
Vázquez, L. B., \& Valenzuela-Galván, D. (2009). ¿Qué tan bien representados están los mamíferos mexicanos en la red federal de áreas naturales protegidas del país? Revista mexicana de Biodiversidad, 80(1), 249-258.

Yañez-Arenas, C., Martínez-Meyer, E., Mandujano, S., \& Rojas-Soto, O. (2012). Modelling geographic patterns of population density of the white-tailed deer in central Mexico by implementing ecological niche theory. Oikos, 121(12), 2081-2089. 